\title{
Sinterização de cerâmicas em microondas. Parte III: Sinterização de zircônia, mulita e alumina
}

\author{
(Microwave sintering of ceramics. \\ Part III: Sintering of zirconia, mullite and alumina)
}

\author{
R. R. Menezes, P. M. Souto, R. H. G. A. Kiminami \\ Laboratório de Desenvolvimento e Processamento de Materiais em Microondas - LaDProM \\ Departamento de Engenharia de Materiais, Universidade Federal de S. Carlos \\ Rod. Washington Luiz, km 235, S. Carlos, SP 13565-905 \\ rrmboca@iris.ufscar.br,ruth@power.ufscar.br
}

\begin{abstract}
Resumo
O aquecimento utilizando microondas possui muitas vantagens com relação aos métodos convencionais de aquecimento, como redução no tempo de processamento, economia de energia e melhora na uniformidade microestrutural dos corpos cerâmicos. Assim esse trabalho tem por objetivo a utilização da sinterização híbrida com microondas na queima rápida de materiais cerâmicos. Foram utilizados materiais que requerem altas temperaturas para densificação. Foram sinterizados materiais que apresentam bruscas e acentuadas mudanças nas suas propriedades dielétricas com a elevação da temperatura, zircônia, e materiais com baixas perdas dielétricas na temperatura ambiente, que apresentam dificuldades de aquecimento com microondas em baixas temperaturas, alumina e mulita. Foi utilizando material susceptor como agente auxiliar de aquecimento. Com base nos resultados obtidos podese concluir que o sistema de sinterização híbrida desenvolvido pode ser utilizado com sucesso na sinterização rápida e uniforme dos materiais estudados, sendo possível a sinterização de zircônia em ciclos de 20 min, mulita em ciclos de 16 min e alumina em ciclos de 40 min.
\end{abstract}

Palavras-chave: microondas, sinterização, cerâmicas.

\begin{abstract}
Thermal processing by microwaves offers several advantages over conventional heating methods, such as shorter processing times, energy savings and improved microstructural homogeneity of ceramic bodies. Thus, this work focused on the fast hybrid microwave sintering of ceramic materials that require high sintering temperatures for densification. The materials studied here were zirconia, which displays abrupt and severe increases in dielectric loss with rising temperature, and alumina and mullite, which show low dielectric losses at ambient temperature and are difficult to microwave at low temperatures. A susceptor was used as an auxiliary heating agent. The results indicate that the sintering system developed here can be used efficiently for the rapid, homogeneous sintering of all the ceramics in question. Zirconia was sintered in 20 min, mullite in 16 min and alumina in 40 min heating cycles. Keywords: microwaves, sintering, ceramics.
\end{abstract}

\section{INTRODUÇÃO}

Nas últimas décadas os fornos de microondas se tornaram um aparelho essencial na maioria das cozinhas. Rápidos ciclos de aquecimento e economia de energia, com relação aos métodos convencionais de cozimento são suas principais características [1]. Apesar do uso de microondas ter se disseminado por todo o mundo, a aplicação dessa tecnologia no processamento de materiais é um desenvolvimento relativamente novo. $\mathrm{O}$ uso de energia de microondas no processamento de materiais tem o potencial de oferecer vantagens similares às observadas quando de sua aplicação no processamento de alimentos [1].

No processamento térmico convencional a energia é transferida para o material através de processos de condução, convecção e radiação para a superfície do material e em seguida, por meio de processos de condução e convecção o calor é conduzido para o interior do material. No processamento com microondas a energia é depositada diretamente no interior do material através de processos de interação molecular e atômica com o campo eletromagnético. Essa diferença na forma de aquecimento propicia várias vantagens ao processamento de materiais usando microondas com relação às técnicas de aquecimento convencionais. Como o aquecimento se dá por interação com o campo eletromagnético, ocorre um aquecimento volumétrico do material, o que permite se trabalhar com altas taxas de aquecimento e reduzir os ciclos de processamento. $\mathrm{O}$ aquecimento seletivo do material a ser processado também permite redução dos gastos energéticos, por não ser necessário o aquecimento de toda a estrutura de isolamento e a mobília dos fornos, secadores, etc. [2,3]. 
A sinterização com microondas é uma técnica que oferece enorme potencial para a fabricação de cerâmicas e compósitos cerâmicos. Vários trabalhos vêm demonstrando a eficiência do uso de microondas nos processos de sinterização [1-4]. Todavia, as necessidades particulares da sinterização de compactos cerâmicos fazem desse processo um dos maiores desafios de aplicação do processamento com microondas [5]. Essas necessidades geralmente incluem alguns ou todos dos seguintes pontos: altas temperaturas, elevadas taxas de aquecimento, distribuição uniforme de temperatura e história térmica equivalente em toda a amostra. Objetivando sinterizar materiais cerâmicos pelo aquecimento direto com microondas pode-se deparar com vários problemas fundamentais. Primeiramente, as características de muitas cerâmicas de pequena absorção de energia de microondas em baixas temperaturas, o que torna o aquecimento inicial difícil. Segundo, instabilidades térmicas podem ocorrer durante o aquecimento, o que leva ao fenômeno de "thermal runaway" descontrolado, provocando o aumento exponencial da temperatura e o aquecimento catastrófico da amostra [5-7]. Isso ocorre em uma grande variedade de materiais cerâmicos, incluindo, $\mathrm{Al}_{2} \mathrm{O}_{3}, \mathrm{SiO}_{2}, \mathrm{Fe}_{3} \mathrm{O}_{4}$, $\beta$-alumina e $\mathrm{ZrO}_{2}[2,5,8,9]$. Finalmente, o gradiente de temperatura característico presente durante o aquecimento volumétrico, que pode levar a severas heterogeneidades de temperaturas no corpo cerâmico. Em vários experimentos e estudos envolvendo sinterização, sistemas de isolamento térmico têm sido usados para minimizar esses gradientes [5]. Todavia, o uso de sistemas de isolamento pode agravar seriamente o problema de "thermal runaway" descontrolado [10], o que fez com que fossem desenvolvidas técnicas de aquecimento híbrido, que combinam o aquecimento com microondas e fontes de aquecimento por infravermelho [4, $8,11,12]$.

A aplicação da energia de microondas para a sinterização rápida de cerâmicas faz com as dificuldades de processamento mencionadas anteriormente se intensifiquem significativamente. Todavia, os benefícios da queima rápida [13-15] fizeram com que vários estudos $[4,8,11,12]$ se dedicassem a pesquisar formas de superar esses obstáculos. A técnica mais eficiente e utilizada para a sinterização rápida de cerâmicas usando microondas é o aquecimento híbrido (do inglês "microwave hybrid heating - MHH", também conhecida como sinterização assistida), independentemente das propriedades dielétricas do material cerâmico.

$\mathrm{O}$ aquecimento híbrido pode ser realizado tanto com o auxílio de um sistema convencional de aquecimento, como resistência ou queimadores de gás, como com a utilização de materiais com altas perdas dielétricas em baixas temperaturas, denominados susceptores. Esses materiais absorvem as microondas e aquecem a amostra a semelhança de uma resistência elétrica utilizada no aquecimento convencional. $\mathrm{O}$ aquecimento híbrido possibilita o aquecimento rápido de materiais transparentes às microondas em baixas temperaturas, bem como suaviza os gradientes térmicos em altas temperaturas, sendo aplicado no processamento de cerâmicas com altas e baixas perdas dielétricas. Assim, esse trabalho tem por objetivo a utilização da sinterização híbrida com microondas, usando materiais susceptores, na queima rápida de materiais cerâmicos.

\section{MATERIAIS E MÉTODOS}

Neste trabalho foram sinterizados materiais cerâmicos que requerem altas temperaturas para densificação $\left(>1400{ }^{\circ} \mathrm{C}\right)$. Os materiais utilizados apresentam características dielétricas peculiares, sendo estudado materiais com baixas perdas dielétricas em temperatura ambiente, alumina e mulita, e material que apresenta alterações bruscas e muito acentuadas nas suas propriedades dielétricas com o aumento de temperatura e que, por isso, requer elevada uniformidade térmica durante a sinterização com microondas, zircônia.

Os materiais utilizados nos processos de sinterização foram os seguintes: alumina A1000SG, 99,8\%, Alcoa Industrial, Chemicals Division, com $\mathrm{D}_{50} \approx 0,6 \mu \mathrm{m}$, (o indicado pelo fabricante é $0,4 \mu \mathrm{m}$ ), e um $\mathrm{D}_{90} \approx 1,9 \mu \mathrm{m}$; mulita SCIMAREC, 99,6\%, SCIMAREC Corporation, com $\mathrm{D}_{50} \approx 1,5 \mu \mathrm{m}, \mathrm{D}_{10} \approx 0,6 \mu \mathrm{m}$ e $\mathrm{D}_{90} \approx 3,0 \mu \mathrm{m}$; zircônia, Zirconia Tosoh TZ-3Y, Tosoh Corporation, com $\mathrm{D}_{50} \approx$ $0,4 \mu \mathrm{m}$, e $\mathrm{D}_{90} \approx 1,4 \mu \mathrm{m}$. Os materiais cerâmicos foram prensados uniaxialmente a $23 \mathrm{MPa}$, em molde com $20 \mathrm{~mm}$ de diâmetro e em seguida, prensados isostaticamente a $200 \mathrm{MPa}$. O diâmetro final das pastilhas situou-se em torno de $19 \mathrm{~mm}$ e sua espessura em torno de $3 \mathrm{~mm}$.

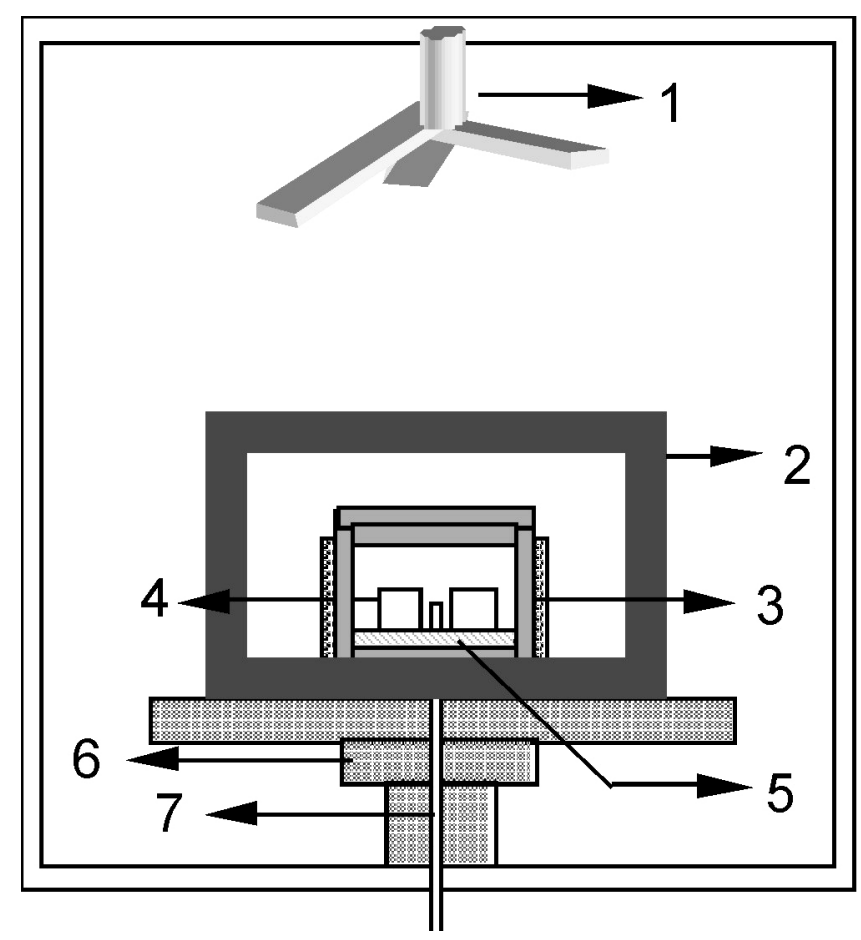

Figura 1: Esquema de sinterização das amostras cerâmicas (fora de proporção): 1) defletor, 2) isolamento, 3) susceptor, 4) cadinho cerâmico, 5) amostra e suporte, 6) mesa rotatória, 7) termopar.

[Figure 1: Scheme of the sintering setup (not on scale): 1) deflector, 2) insulator, 3) susceptor, 4) ceramic crucible, 5) sample and holder, 6) rotating table, 7) thermocouple.] 
As pastilhas foram submetidas a processos de sinterização em forno de microondas (Cober Electronics, modelo MS6K), a 2,45 GHz, utilizando material susceptor como agente auxiliar de aquecimento para a sinterização híbrida. A Fig. 1 ilustra o esquema utilizado para a sinterização das amostras. A fim de propiciar a comparação dos resultados as amostras também foram sinterizadas em forno convencional.

As amostras de zircônia foram submetidas a sinterização em forno convencional a $1450{ }^{\circ} \mathrm{C}$ e $1500{ }^{\circ} \mathrm{C}$ por $2 \mathrm{~h}$, com taxa de aquecimento de $5{ }^{\circ} \mathrm{C} / \mathrm{min}$ e em forno de microondas por 16 e 20 min com potência fixa de irradiação de $1,8 \mathrm{~kW}$. As amostras de mulita foram sinterizadas em forno convencional $1500{ }^{\circ} \mathrm{C}, 1550{ }^{\circ} \mathrm{C}, 1600{ }^{\circ} \mathrm{C}$ e $1650{ }^{\circ} \mathrm{C}$ por $2 \mathrm{~h}$, em alto vácuo, com um taxa de aquecimento de $15^{\circ} \mathrm{C} / \mathrm{min}$ e em forno de microondas por 16 min com potência fixa de irradiação de $1,5 \mathrm{~kW}$. As amostras de alumina foram sinterizadas em forno convencional a $1550{ }^{\circ} \mathrm{C}$ e $1600{ }^{\circ} \mathrm{C}$ por $2 \mathrm{~h}$, com taxa de aquecimento de $5{ }^{\circ} \mathrm{C} / \mathrm{min}$ e em forno de microondas por vários tempos de queima e potência fixa de irradiação de 1,8 $\mathrm{kW}$. Visando avaliar o sistema de controle de aquecimento para altas temperaturas desenvolvido no LaDProM, algumas amostras de alumina também foram submetidas a ciclos de aquecimento com controle de temperatura, utilizando-se taxas de aquecimento de $100{ }^{\circ} \mathrm{C} / \mathrm{min}$ até $1100{ }^{\circ} \mathrm{C}$ e de $50{ }^{\circ} \mathrm{C} / \mathrm{min}$ a partir de então.

As densidades das amostras sinterizadas foram determinadas pelo método de Archimedes com imersão em água e as suas microestruturas foram analisadas por microscopia eletrônica de varredura, MEV (Phillips, XL30 FEG).

\section{RESULTADOS E DISCUSSÃO}

A Tabela I apresenta os resultados de densificação das amostras de zircônia sinterizadas em microondas por 16 e 20 min, e também os resultados obtidos com a sinterização convencional. Com base nos resultados de densidade (Tabela I) obtidos com a sinterização convencional, verifica-se que ciclos de queima de apenas 20 min em microondas podem conduzir a densidades superiores às obtidas em ciclos de queima de 2 h a $1450{ }^{\circ} \mathrm{C}$. Os resultados de densificação observados com a sinterização convencional a $1450{ }^{\circ} \mathrm{C}$ são inferiores a valores observados na literatura, o que pode ser consequiência do processamento do pó do material (as amostras compactadas atingiram densidades a verde em torno de 47\%). No entanto, e mais importante, é que com os resultados obtidos com a sinterização híbrida em microondas passa a haver um indicativo de que o uso de microondas, no caso da zircônia, pode conduzir amostras de zircônia a altas densificações, mesmo partindo-se de baixas densidades a verde, o que, geralmente não se observa com a sinterização convencional.

As Figs. 2 e 3 apresentam a micrografia e a distribuição do tamanho de grãos da amostra Z1,8kW20min-M, que foi sinterizada em forno de microondas por $20 \mathrm{~min}$ sob potência fixa de irradiação de $1,8 \mathrm{~kW}$. A amostra apresentou tamanho médio de grão de $0,6 \pm 0,3 \mu \mathrm{m}$, o que indica que ocorreu um crescimento de grão muito pequeno. Deve-se ressaltar, que pode ter ocorrido crescimento de grão durante o ataque térmico da amostra

A zircônia possui propriedades dielétricas que se alteram muito acentuadamente e rapidamente com o aumento da temperatura, fazendo com que qualquer heterogeneidade térmica durante sua sinterização em microondas possa conduzir à formação de trincas e comprometimento estrutural do material. Esse comportamento faz com que seja necessário o controle da homogeneidade térmica na peça durante o processo de aquecimento para se ter sucesso na sua sinterização. Em virtude dessa necessidade de homogeneidade térmica, a sua queima em microondas (na freqüência de $2,45 \mathrm{GHz}$ ) só é possível utilizando-se sistemas susceptores que auxiliem no aquecimento e na homogeneização da temperatura na amostra. Um estudo abordando a sinterização de zircônia tetragonal estabilizada com ítria [16] relatou a sinterização sem a utilização de susceptores; entretanto muito tempo foi gasto para otimizar o sistema de isolamento, foram realizadas várias tentativas de sinterização antes de se obter algum sucesso e, mesmo assim, uma grande quantidade de peças (pelos menos 15\%) se quebrava ou desenvolvia trincas durante a sinterização.

Dentre os demais estudos observados na literatura que abordaram a sinterização da zircônia (todos utilizando susceptores), muito poucos $[17,18]$ conseguiram sinterizações, com densificação das amostras de TZ-3Y (Tosoh) acima de $95 \%$, em tempos inferiores a $45 \mathrm{~min}$. Observase que em geral os trabalhos abordando a sinterização de zircônia estabilizada em microondas conseguem densificações acima de $98 \%$, sem grande crescimento de grão (obtendo-se tamanhos sub micrométricos); entretanto, o tempo de sinterização é superior a $60 \mathrm{~min}$, independentemente da sinterização ter sido realizada controlando-se a taxa de aquecimento ou apenas fixandose uma potência de irradiação para todo o processo. Esse elevado tempo de sinterização em microondas, não é uma conseqüência apenas de limitações de potências dos equipamentos, mas do comportamento de aquecimento da zircônia e das limitações dos arranjos susceptores utilizados [11, 19-21]. Nesse estudo, a utilização de um sistema otimizado de aquecimento auxiliar, possibilitou a sinterização híbrida com microondas de amostras de zircônia em ciclos de aquecimento significativamente pequenos, que não ultrapassaram $20 \mathrm{~min}$.

Tabela I - Densidade e tamanho médio de grãos de amostras de zircônia sinterizadas em forno convencional e em forno de microondas.

[Table I - Density and average grain size of zirconia samples sintered in conventional and microwave ovens.]

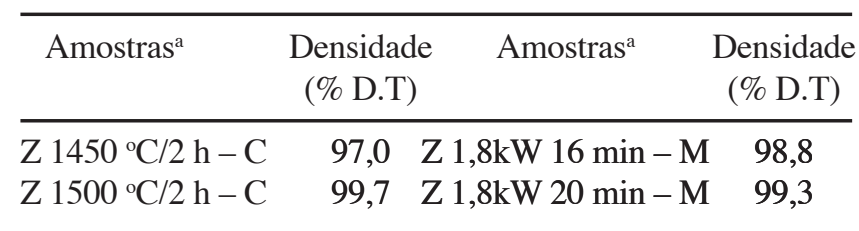

aletra C designa sinterização em forno convencional e a letra $M$ em forno de microondas 


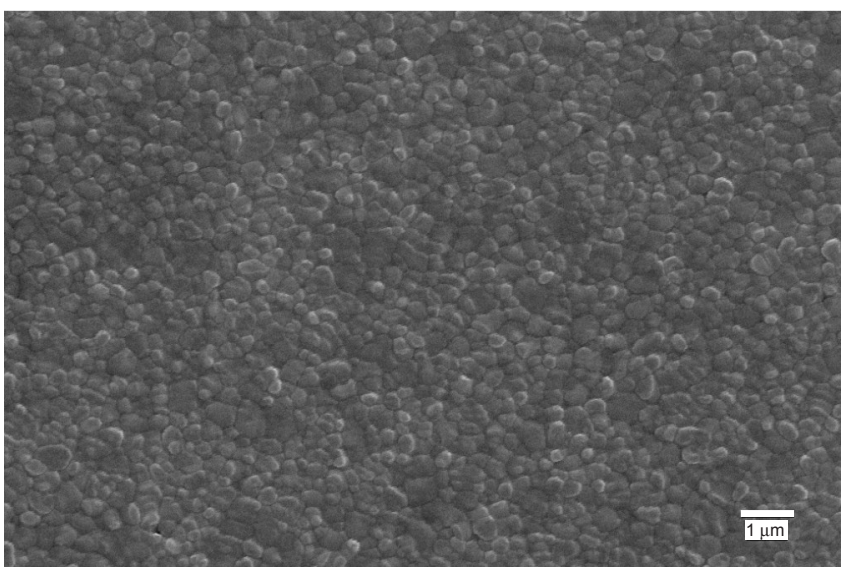

Figura 2: Micrografia da amostra de zircônia Z 1,8kW 20min, sinterizada em forno de microondas.

[Figure 2: SEM micrograph of the Z $1.8 \mathrm{~kW} 20$ min zirconia sample, sintered in microwave oven.]

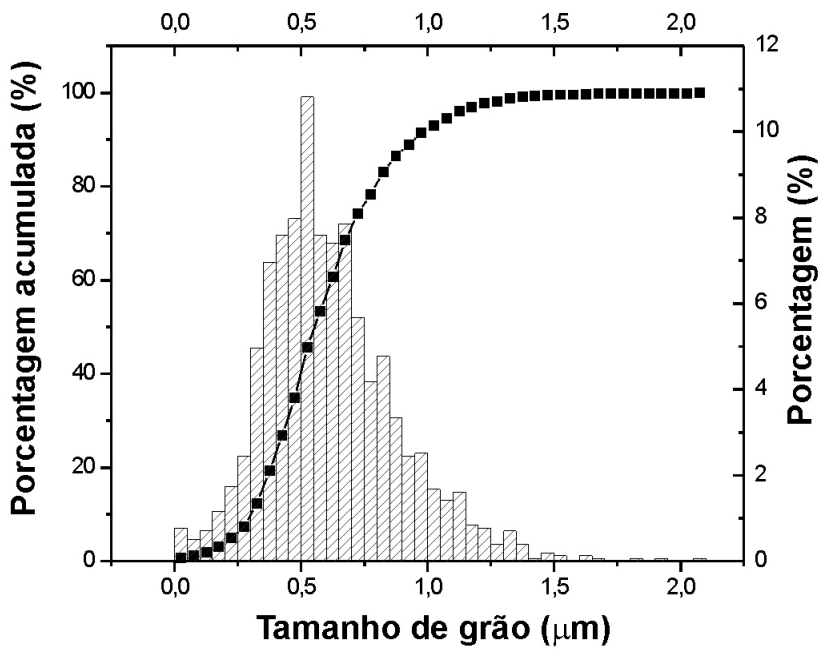

Figura 3: Distribuição de tamanho de grão da amostra de zircônia Z $1,8 \mathrm{~kW} 20 \mathrm{~min}$, sinterizada em forno de microondas.

[Figure 3: Grain size distribution of the Z $1.8 \mathrm{~kW} 20$ min zirconia sample, sintered in microwave oven.]

Apesar dos rápidos ciclos de queima (altas taxas de aquecimento) não foram observados em nenhuma amostra trincas ou danos decorrentes de heterogeneidades no aquecimento híbrido e/ou formação de "hot spots".

A Fig. 4 apresenta as densidades obtidas com a sinterização convencional em alto vácuo e a sinterização híbrida com microondas das amostras de mulita. Pode-se observar que a densificação dos compactos só é observada em altas temperaturas, o que está provavelmente associado ao elevado tamanho de partícula do pó de mulita $(\sim 1,5 \mu \mathrm{m})$, à sua morfologia, que dificulta a densificação a verde dos compactos [9] e ao baixo coeficiente de difusão do Si na rede cristalina da mulita. Por outro lado, a mulita também apresenta baixas perdas dielétricas na temperatura ambiente [22], o que, associado a sua característica de difícil densificação, faz com que seja complicada a sua densificação através de processos

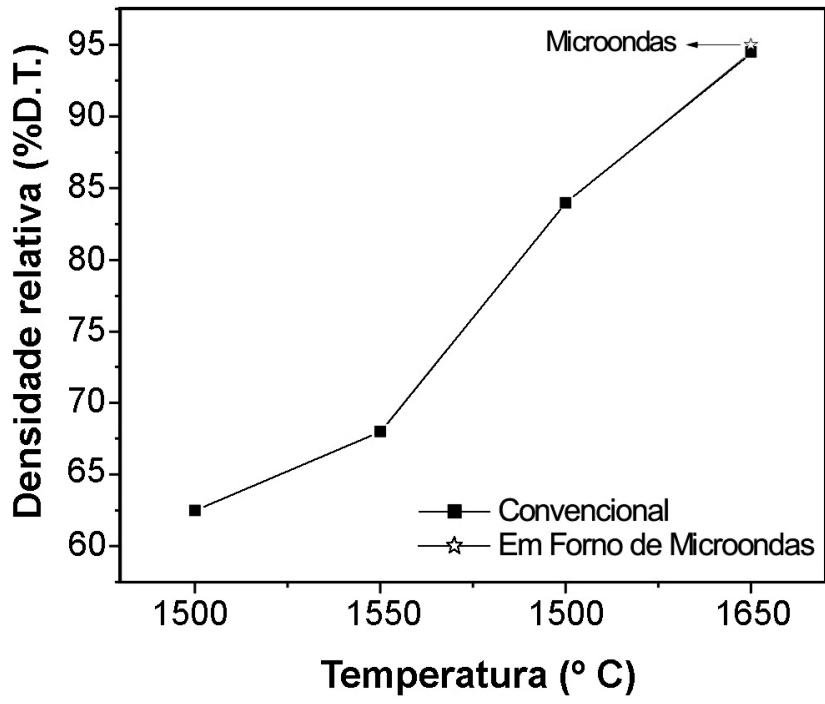

Figura 4: Densidades relativas das amostras de mulita sinterizadas convencionalmente (em alto vácuo) e em forno de microondas.

[Figure 4: Relative density of the mullite samples after conventional sintering (under high vacuum) and in microwave oven.]
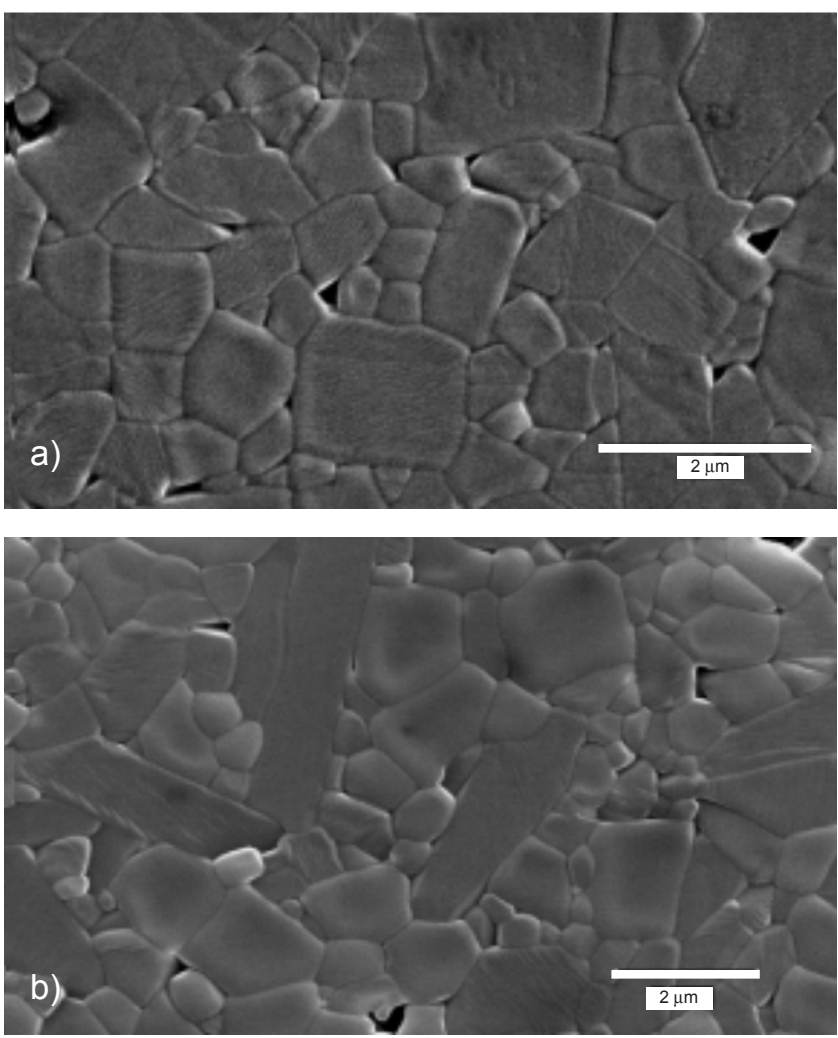

Figura 5: Micrografias das amostras de mulita sinterizadas em: a) forno de microondas, $1,5 \mathrm{~kW}-16 \mathrm{~min}, \mathrm{~b}$ ) convencional (alto vácuo) a $1650{ }^{\circ} \mathrm{C} / 2 \mathrm{~h}$.

[Figure 5: SEM micrographs of mullite samples sintered in: a) microwave oven, $1.5 \mathrm{~kW}-16 \mathrm{~min}$, b) conventional (high vacuum) at $\left.1650^{\circ} \mathrm{C} / 2 \mathrm{~h}.\right]$

de sinterização com o uso de microondas. Entretanto, foi possível sinterizar em microondas os compactos de mulita em ciclo de $16 \mathrm{~min}$, (potência de 1,5 kW), atingindo-se densidade de $95 \%$. A mesma densidade só foi atingida no 


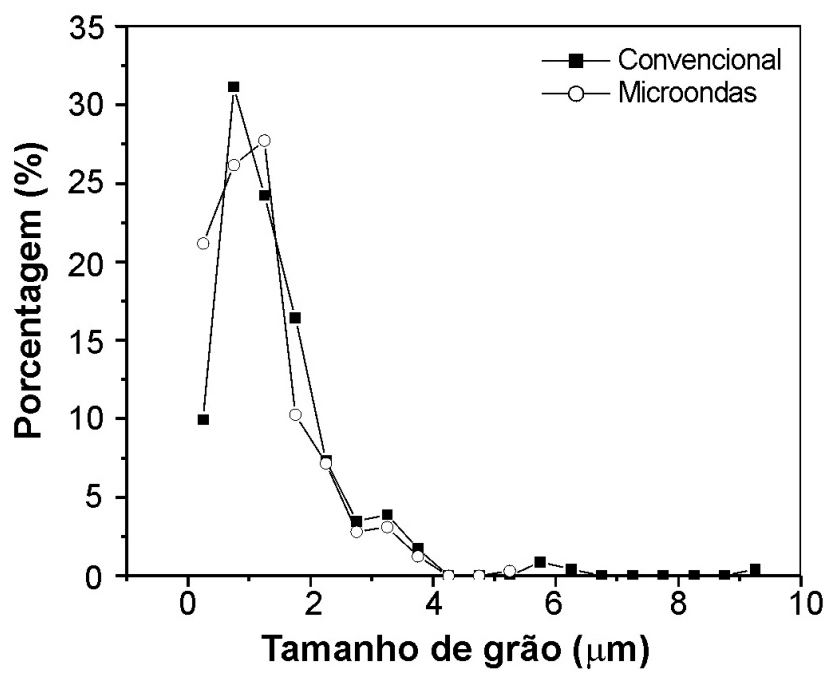

Figura 6: Distribuição de tamanho de grãos das amostras de mulita sinterizadas em forno convencional (em alto vácuo) e em forno de microondas.

[Figure 6: Grain size distribution of mullite samples sintered in conventional (high vacuum) and microwave ovens.]

ciclo de queima convencional a $1650^{\circ} \mathrm{C} / 2 \mathrm{~h}$.

As Figs. 5 e 6 apresentam as micrografias e as distribuições de tamanho de grãos das amostras de mulita sinterizadas em forno de microondas e em forno convencional. A mulita sinterizada convencionalmente apresentou tamanho médio de grão de $1,41 \pm 1,07 \mu \mathrm{m}$ e a sinterizada em microondas apresentou um tamanho médio de $1,18 \pm 0,82$ $\mu \mathrm{m}$. Entretanto, pode-se observar através da Fig. 5, que apesar dos tamanhos médios de grão serem semelhantes, a distribuição dos tamanhos de grãos da mulita sinterizada em forno convencional é mais larga que à observada para a mulita sinterizada em microondas, apresentando grãos com dimensões, de até oito vezes o tamanho médio (um crescimento quase abnormal de grão).

A maioria da literatura [23-27] envolvendo a sinterização em microondas de corpos de mulita estuda a sinterização de nanopós, de compósitos a base de mulita ou aborda processos envolvendo a síntese e sinterização simultâneas de corpos mulíticos a partir de precursores orgânicos e inorgânicos. Assim, torna-se difícil a comparação dos resultados obtidos com outros dados. Todavia, frente aos resultados da sinterização convencional observa-se de imediato um grande potencial econômico no uso da sinterização híbrida com microondas na densificação da mulita, bem como, vantagens tecnológicas, em virtude da maior homogeneidade microestrutural dos corpos sinterizados utilizando energia de microondas.

A Fig. 7 e a Tabela II apresentam as densidades relativas das amostras de alumina sinterizadas em forno convencional e em forno de microondas. Pode-se observar que tempos de sinterização de 35 min são suficientes para se obter densidades semelhantes às obtidas com a sinterização convencional a $1600{ }^{\circ} \mathrm{C} / 2 \mathrm{~h}$. Observa-se também que a sinterização com microondas por $35 \mathrm{~min}$ conduz a valores superiores aos obtidos com a queima em forno convencional
Tabela II - Densidade e tamanho médio de grãos de amostras de alumina sinterizadas em forno convencional e em forno de microondas.

[Table II - Density and average grain size of alumina samples sintered in conventional and microwave ovens.]

\begin{tabular}{|c|c|c|c|}
\hline \multirow[t]{2}{*}{ Amostras $^{\mathrm{a}}$} & \multirow[t]{2}{*}{$\begin{array}{c}\text { Densidade } \\
\text { (\% D.T.) }\end{array}$} & \multicolumn{2}{|c|}{$\begin{array}{c}\text { Tamanho de } \\
\text { Grão } \pm \text { Desvio } \\
\text { Padrão }(\mu \mathrm{m})\end{array}$} \\
\hline & & Interior & Extremidade \\
\hline A 15 & 96,7 & \multicolumn{2}{|c|}{$2,2 \pm 1,6$} \\
\hline A $1600{ }^{\circ} \mathrm{C} / 2 \mathrm{~h}$ & 97,6 & \multicolumn{2}{|c|}{$4,2 \pm 2,9$} \\
\hline A $1,8 \mathrm{~kW} 30 \mathrm{~min}-\mathrm{M}$ & 96,6 & $2,8 \pm 1,9$ & $2,5 \pm 1,9$ \\
\hline A $1,8 \mathrm{~kW} 35 \mathrm{~min}-\mathrm{M}$ & 97,5 & & 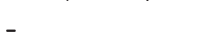 \\
\hline A $1,8 \mathrm{~kW} 40 \mathrm{~min}-\mathrm{M}$ & 97,9 & $4,1 \pm 3,8$ & $3,7 \pm 3,4$ \\
\hline
\end{tabular}

aletra $C$ designa sinterização em forno convencional e a letra $M$ em forno de microondas

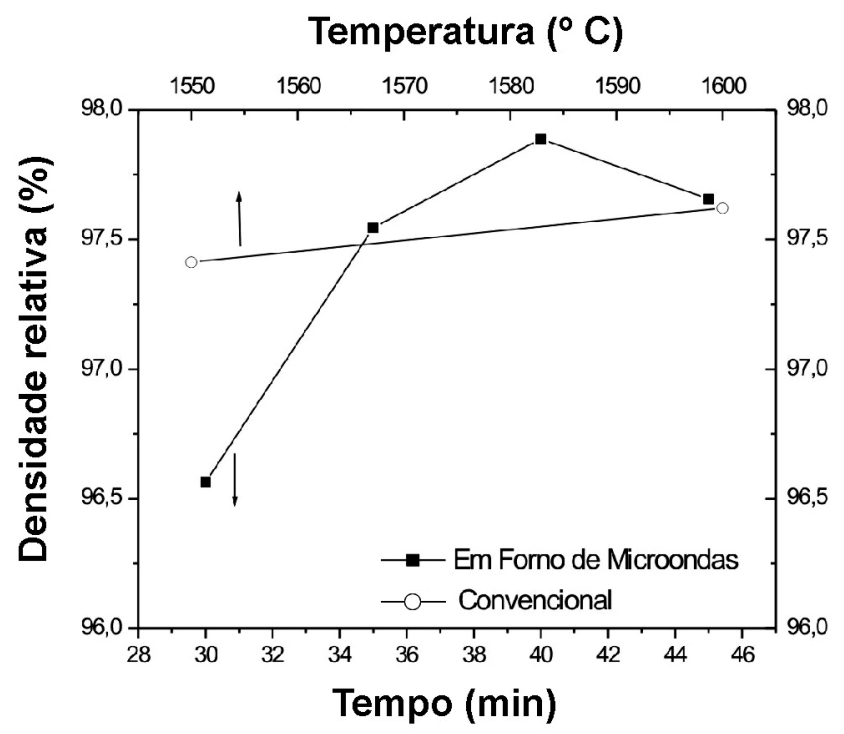

Figura 7: Densidades relativas das amostras de alumina sinterizadas convencionalmente e em forno de microondas. [Figure 7: Relative density of alumina samples after conventional and microwave sintering.]

e que, o aumento do tempo de sinterização em microondas aparenta provocar a diminuição da densidade. Vale salientar, que os baixos valores de densidade obtidos provavelmente estão associados à larga distribuição de tamanho de partículas da matéria-prima utilizada e a questões de processamento, já que foram observados tanto na sinterização convencional como na híbrida com microondas.

As Figs. 8 e 9 apresentam as micrografia e a distribuição do tamanho de grãos da amostra A1,8kW40min-M, que foi sinterizada em forno de microondas por $40 \mathrm{~min}$ sob potência fixa de irradiação de $1,8 \mathrm{~kW}$. Pode-se observar que não ocorreram heterogeneidades microestruturais associadas a diferentes distribuições de tamanho de grão no interior e na região próxima a superfície da amostra. 

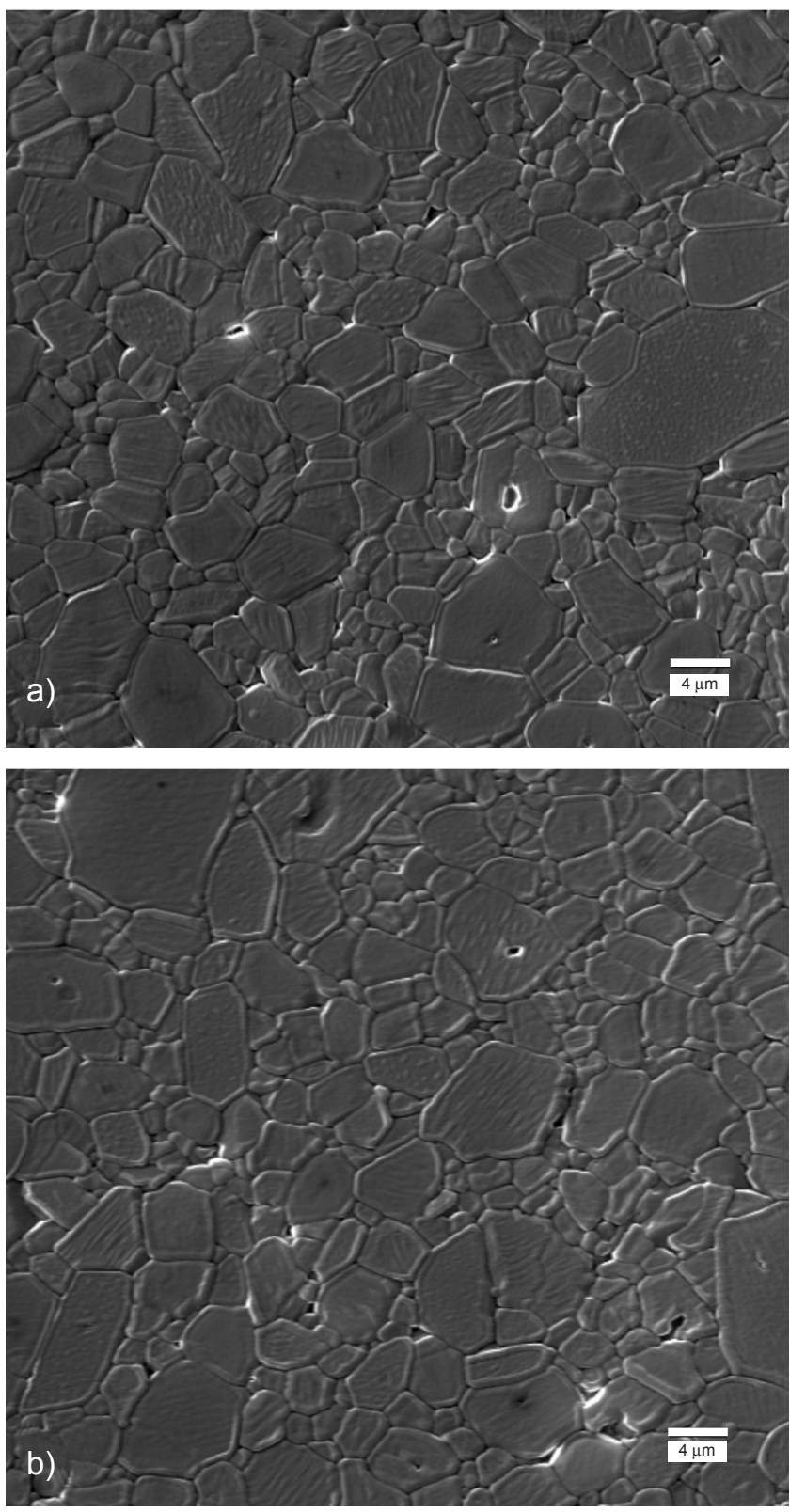

Figura 8: Micrografias de amostra de alumina (A1,8 kW 40 min-M) sinterizada em forno de microondas: a) região interior da amostra e b) região próxima a superfície da amostra.

[Figure 8: SEM micrographs of an alumina sample (A $1.8 \mathrm{~kW} 40$ min-M) sintered in microwave oven: a) inside the sample and $b$ ) close to the surface.]

Verifica-se, que apesar do elevado desvio padrão, o tamanho médio dos grãos no interior da amostra é praticamente igual ao observado na região próxima à superfície, o que evidencia que o sistema susceptor foi bem sucedido em suavizar gradientes térmicos presentes nas amostras em altas temperaturas e que não ocorreu apenas o aquecimento indireto da amostra (semelhantemente a um aquecimento convencional).

Como mencionado anteriormente, cerâmicas transparentes às microondas são extremamente complicadas de aquecer em baixas temperaturas sem o auxílio de fontes externas de aquecimento, o que dificulta a sua sinterização

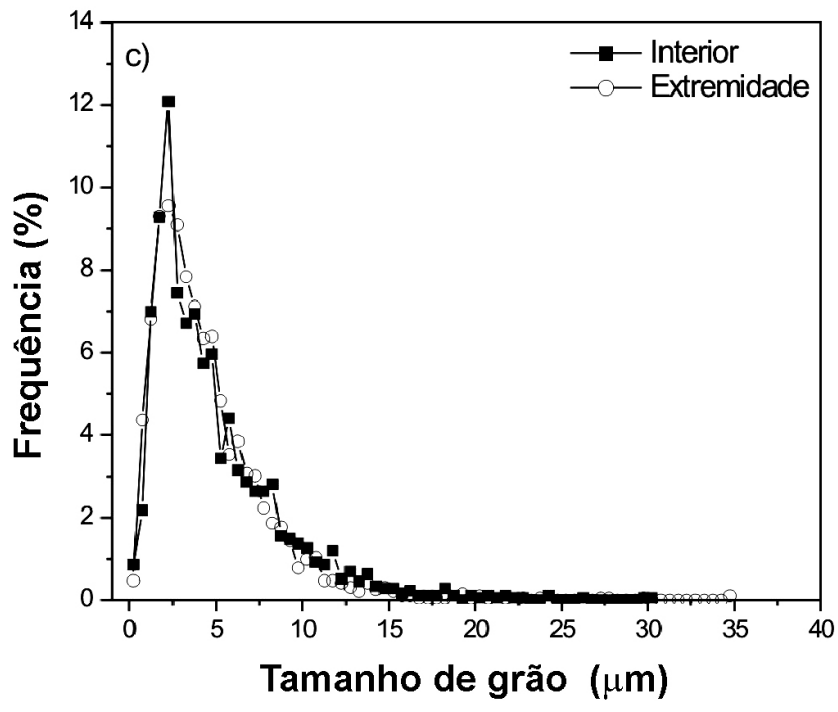

Figura 9: Distribuição do tamanho de grão de amostra de alumina (A 1,8 kW 40 min-M) sinterizada em forno de microondas.

[Figure 9: Grain size distribution of an alumina sample (A $1.8 \mathrm{~kW}$ 40 min- M) sintered in microwave oven.]

com a utilização de microondas, particularmente quando se deseja trabalhar com ciclos de queima rápida. O processamento de cerâmicas transparentes com elevadas taxas de aquecimento requer que a fonte de aquecimento auxiliar, no nosso caso o material susceptor, tenha alta capacidade de aquecimento nos estágios iniciais do processo e, ao mesmo tempo, possibilite uma homogeneização nas temperaturas do corpo cerâmico em altas temperaturas, a fim de evitar o desenvolvimento de heterogeneidades microestruturais no material.

As heterogeneidades microestruturais em uma queima rápida utilizando microondas e aquecimento híbrido, podem surgir tanto por uma resposta muito rápida do susceptor, não acompanhada pela amostra (à semelhança de uma queima rápida convencional), quanto por consequiência da interação intensa da amostra com a radiação e elevação da sua temperatura rapidamente, sem ocorrer um aquecimento similar por parte do susceptor. Pode-se observar ambos tipos de comportamento na sinterização rápida de alumina utilizando-se aquecimento híbrido com microondas [4, 12]. No entanto, mesmo utilizando-se rápidos ciclos de queima, não foi observado no presente trabalho heterogeneidades microestruturais nas amostras de alumina sinterizadas utilizando-se microondas.

Comparativamente a sinterização convencional, observase que o tamanho de grão das amostras sinterizadas usando microondas foram praticamente iguais (analisando em densidades semelhantes), não sendo observado refinamento da microestrutura, mesmo em curtos ciclos de queima.

A fim de avaliar o desempenho de aquecimento do sistema de controle de temperatura desenvolvido no LaDProM para altas temperaturas (em trabalho anterior [28], foi observado seu excelente desempenho em sinterizações utilizando baixas temperaturas, $<1200^{\circ} \mathrm{C}$ ), amostras de alumina foram 
submetidas a queima rápida utilizando aquecimento híbrido com microondas. Inicialmente observou-se que as curvas monitoradas de temperatura apresentavam defasagens em relação às pré-programadas quando se trabalhava com taxas de aquecimento superiores a $75^{\circ} \mathrm{C} / \mathrm{min}$ e até temperaturas da ordem de $700{ }^{\circ} \mathrm{C}$. A partir de então, a diferença de temperatura não superava $5{ }^{\circ} \mathrm{C}$, mesmo com taxas superiores a $75^{\circ} \mathrm{C} / \mathrm{min}$. Nas temperaturas de patamar essas diferenças não ultrapassavam $3{ }^{\circ} \mathrm{C}$. Acredita-se que esse comportamento está relacionado à inércia térmica do sistema, já que a defasagem existia abaixo dos $700{ }^{\circ} \mathrm{C}$; no entanto, a taxa de
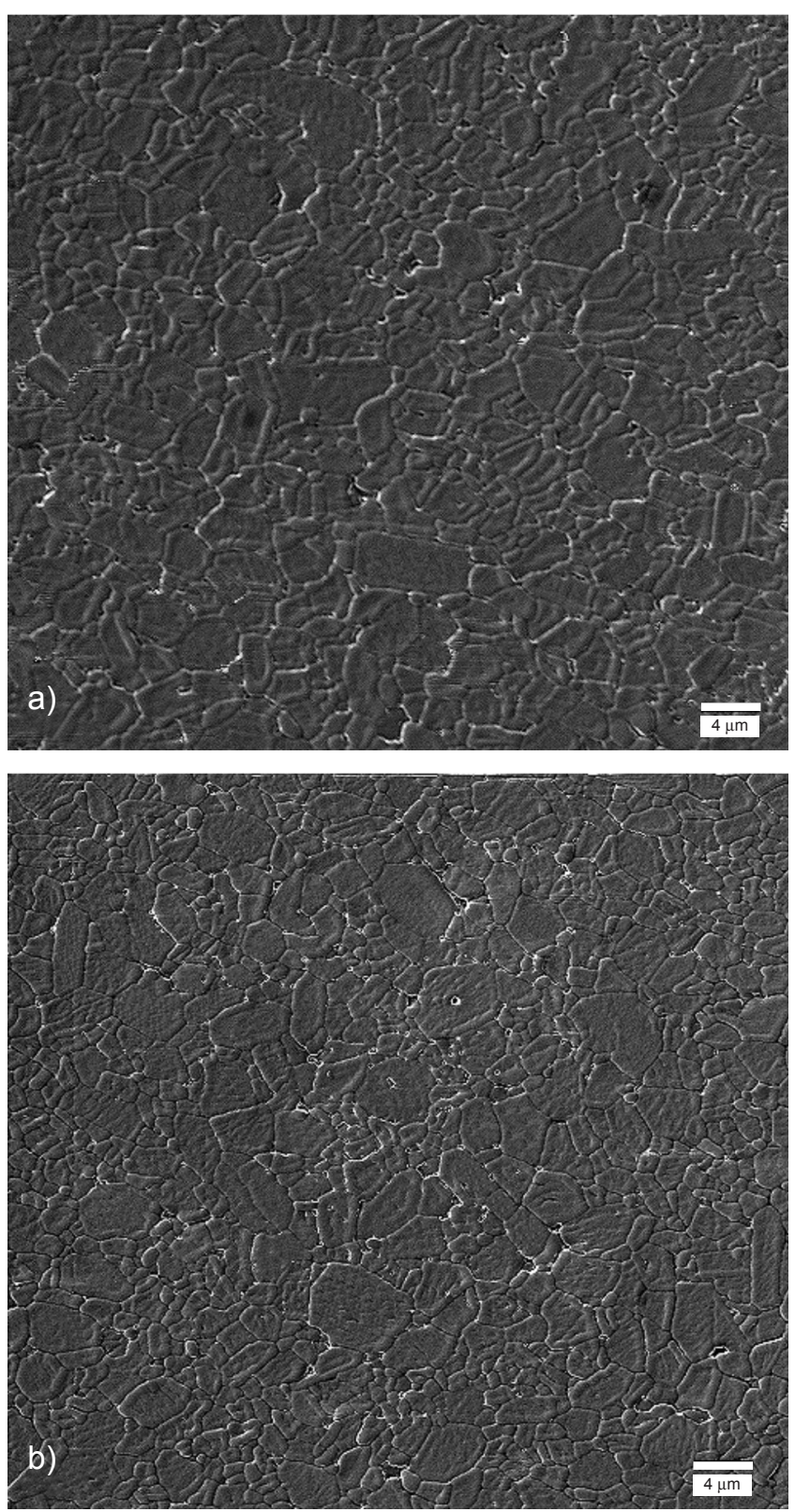

Figura 10: Micrografias de amostra de alumina sinterizada em forno de microondas a $1550{ }^{\circ} \mathrm{C} / 2 \mathrm{~h}$ : a) região interior da amostra e b) região próxima a superfície da amostra.

[Figure 10: SEM micrographs of an alumina sample sintered in microwave oven at $1550{ }^{\circ} \mathrm{C} / 2 \mathrm{~h}$ : a) inside the sample and b) close to the surface.]

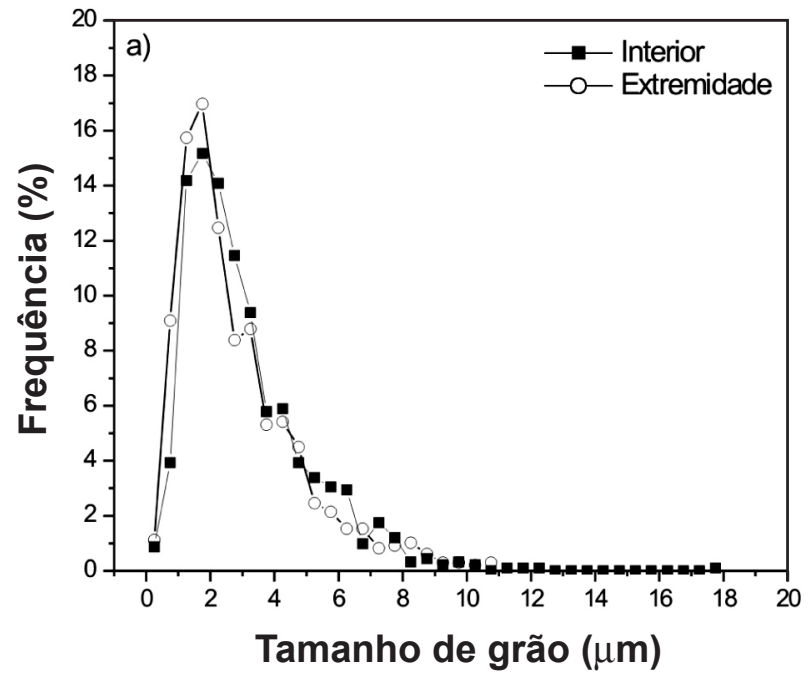

Figura 11: Distribuição do tamanho de grão de amostra de alumina sinterizada em forno de microondas a $1550{ }^{\circ} \mathrm{C} / 2 \mathrm{~h}$.

[Figure 11: Grain size distribution of an alumina sample sintered in microwave oven at $1550^{\circ} \mathrm{C} / 2 \mathrm{~h}$.]

aquecimento, a partir dos $400-450^{\circ} \mathrm{C}$, era igual à programada ou a superava, quando a defasagem de temperaturas atingia valores superiores a $30-35^{\circ} \mathrm{C}$, a fim de eliminar a diferença na temperatura programada e na real. Com a otimização do sistema susceptor passou-se a ser possível a utilização de taxas de aquecimento superiores a $100{ }^{\circ} \mathrm{C} / \mathrm{min}$, mesmo em baixas temperaturas, sem problemas de elevadas defasagens nos estágios iniciais de aquecimento.

Nesse sentido, a utilização de susceptores capazes de aquecer rapidamente em baixas temperaturas é fundamental, a fim de se evitar a necessidade de uso de potências elevadas em baixas temperaturas, o que pode provocar a ionização do termopar.

Amostras de alumina foram sinterizadas com sucesso a $1550{ }^{\circ} \mathrm{C}$ por $30 \mathrm{~min}$, utilizando-se taxas de aquecimento de $100{ }^{\circ} \mathrm{C} / \mathrm{min}$ até $1100{ }^{\circ} \mathrm{C}$ e de $50{ }^{\circ} \mathrm{C} / \mathrm{min}$ até $1550{ }^{\circ} \mathrm{C}$. Obteve-se uma densidade de 96,8\% (\%.D.T.). As Figs. 10 e 11 apresentam as micrografias e a distribuição de tamanho de grão das amostras sinterizadas. Pode-se observar que houve uma distribuição de grão semelhante no interior da amostra e na região próxima a superfície, o que evidencia que o sistema de sinterização híbrida com microondas utilizando curva de aquecimento pré-programada possibilita aquecer peças cerâmicas em altas temperaturas de forma homogênea, apesar das elevas perdas térmicas que ocorrem em temperaturas mais elevadas. $\mathrm{O}$ tamanho médio de grão obtido foi de 3,1 $\pm 1,9 \mu \mathrm{m}$ no interior da amostra e de 2,8 \pm $1,9 \mu \mathrm{m}$ na região próxima a superfície da amostra.

Analisando os resultados obtidos com relação aos da sinterização convencional e a de microondas realizada com potência fixa (Tabela II), observa-se que o tamanho de grão é ligeiramente superior ao obtido com a sinterização por 30 min a $1,8 \mathrm{~kW}$, cujas amostras apresentaram densidade relativa semelhante à obtida com a queima a $1550{ }^{\circ} \mathrm{C}$ por $30 \mathrm{~min}$ em microondas. Com relação a sinterização convencional, 
observa-se que o tamanho de grão obtido com a sinterização a $1550{ }^{\circ} \mathrm{C}$ por $30 \mathrm{~min}$ em microondas é superior àquele obtido com a sinterização a $1550^{\circ} \mathrm{C}$ por $2 \mathrm{~h}$, mesmo tendo-se obtido uma densidade semelhante e utilizado-se um tempo de processamento significativamente inferior. Isso pode está associado a uma possível intensificação da cinética de difusão, uma manifestação do "efeito microondas" [29], que favoreceu o crescimento dos grãos, em virtude do largo tempo de exposição às microondas.

Os resultados apresentados evidenciam a eficiência do sistema de sinterização híbrida com microondas desenvolvido no LaDProM na sinterização rápida e uniforme de cerâmicas com baixas perdas dielétricas na temperatura ambiente, transparentes às microondas, e na sinterização de cerâmicas com propriedades dielétricas complexas, que se alteram rápida e acentuadamente com o aumento da temperatura.

\section{CONCLUSÕES}

Este trabalho teve como objetivo a utilização da sinterização híbrida com microondas na queima rápida de materiais cerâmicos. Com base nos resultados obtidos podese concluir que:

- o sistema de sinterização híbrida com microondas pode ser utilizado com sucesso na sinterização rápida e uniforme de zircônia, alumina e mulita, independentemente das características particulares de sinterização em microondas desses materiais;

- o sistema desenvolvido possibilita ciclos de processamento semelhantes e por vezes inferiores, aos observados na literatura internacional abordando $\mathrm{o}$ aquecimento rápido de cerâmicas com microondas;

- é possível a sinterização rápida e uniforme de materiais que apresentam variações bruscas e acentuadas das suas propriedades dielétricas com o aumento da temperatura, como zircônia, em ciclos de aquecimento de $20 \mathrm{~min}$, sem a ocorrência de "thermal runaway" descontrolado e/ou desenvolvimento de trincas nas amostras;

- é possível a sinterização rápida e uniforme de materiais transparentes as microondas na temperatura ambiente, como alumina e mulita, em ciclos de aquecimento inferiores a $40 \mathrm{~min}$.

\section{AGRADECIMENTOS}

À FAPESP (05/60095-6, 06/50121-5) e ao CNPq (476382/2004-1) pelo apoio financeiro.

\section{REFERÊNCIAS}

[1] E. T. Thostenson, T. W. Chou, Composites: Part A 30 (1999) 1055.

[2] W. H. Sutton, Am. Ceram. Soc. Bull. 68, 2 (1989) 376.

[3] D. E. Clark, W. H. Sutton, Annu. Rev. Mater. Sci. 26 (1996) 299.

[4] A. Dé, I. Ahmad, D. Whitney, D. E. Clark, Ceramic Transactions, Microwaves: Theory and applications in materials processing, Eds.: D. E. Clark, F. D. Gac, W. H. Sutton, American Ceramic Society, Westerville, Vol. 21 (1991) 319.

[5] M. S. Spotz, D. J. Skamser, D. L. Johnson, J. Am. Ceram. Soc. 78, 4 (1995) 1041.

[6] A. J. Berteaud, J. C. Badot, J. Microwave Power 11, 4 (1976) 315.

[7] G. Roussy, A. Bennani, J. M. Thiebaut, J. Appl. Phys. 62, 4 (1987) 1167.

[8] M. A. Janney, C. L. Calhoun, H. D. Kimrey, J. Am. Ceram. Soc. 75, 2 (1992) 341.

[9] R. R. Menezes, P. M. Souto, E. Fagury-Neto, R. H. G. A. Kiminami, Proc. Fourth World Congress on Microwave and Radio Frequency Application, Eds.: R. L Shulz and D. C. Folz. The Microwave Working Group, Arnold, MD (2004) 118

[10] D. L. Johnson, D. J. Skamser, M. S. Spotz, Microwaves: Theory and applications in materials processing II, Eds: J. Binner, D. Whitaker, American Ceramic Society, Westerville, Vol. 36 (1993) 133.

[11] M. A. Janney, C. L. Calhon, H. D. Kimrey, J. Am. Ceram. Soc. 75, 2 (1992) 341.

[12] A. Dé, I. Ahmad, D. Whitney, D. E. Clark, Ceramic transactions, Microwaves: Theory and applications in materials processing, Eds.: D. E. Clark, F. D. Gac, W. H. Sutton, American Ceramic Society, Westerville, Vol. 21 (1991) 329.

[13] M. P. Harmer, E. W. Roberts, R. J. Brook, Trans. J. Br. Ceram. Soc. 78 (1979) 22.

[14] M. P. Harmer, R. J. Brook, Trans. Br. Ceram. Soc. 80, 5 (1981) 147.

[15] T. Manfredini, L. Pennisi, Ceramic transactions, Science of Whitewares, Eds.: V. E. Henkes, G. Y. Onoda, W. M. Carty, American Ceramic Society, Westville, Ohio, (1996) 213.

[16] A. Goldstein, N. Travitzky, A. Singurindy, M. Kravchik, J. Eur. Ceram. Soc. 19 (1999) 2067.

[17] J. Wilson, S. M. Kunz, J. Am. Ceram. Soc. 71, 1 (1988) C40.

[18] D. Upadhyaya, A. Ghosh, G. K. Dey, R. Prasad, A. K. Suri, J. Mater. Sce. 36 (2001) 4707.

[19] S. A. Nightingale, H. K. Worner, D. P. Dunne, J. Am. Ceram. Soc. 80, 2 (1997) 394.

[20] Z. Xie, J. Yang, X. Huang, Y. Huang, J. Eur. Ceram. Soc. 19 (1999) 381.

[21] S. Fujitsu, M. Ikegami, T. Hayashi, J. Am. Ceram. Soc. 83, 8 (2000) 2085.

[22] W. Xi, W. R. Tinga, Ceramic transactions, Microwaves: Theory and applications in materials processing, Eds.: D. E. Clark, F. D. Gac, W. H. Sutton, American Ceramic Society, Westerville, Vol. 21 (1991) 215.

[23] Y. Fang, Y. Chen, M. R. Silsbee, D. Agrawal, Mater Lett. 27, 4-5 (1996) 155.

[24] Y. Fang, R. Roy, D. K. Agrawal, D. M. Roy, Mater Lett. 28, 1-3 (1996) 11.

[25] B. G. Ravi, V. Praveen, M. P. Selvam, Mater. Res. Bull. 33, 10 (1998) 1527. 
[26] J. Raabe, E. Bobryk, V. Petrovsky, Ceram Int. 27, 1 (2001) 81.

[27] M. Panneerselvam, K. J. Rao, Chemistry of Materials 15, 11 (2003) 2247.

[28] R. R. Menezes, P. M. Souto, R. H. G. A. Kiminami,
Cerâmica 53, 326 (2007) 108-115.

[29] M. A. Janney, H. D. Kimrey, W. R. Allen, J. O. Kiggans, M. A. Janney, H. D. Kimrey, W. R. Allen, J. O. Kiggans, J. Mater. Sci. 32 (1997) 1347.

(Rec. 01/09/2006, Ac. 24/11/2006) 